\title{
IMPACTO DEL CAMBIO CLIMÁTICO SOBRE LOS INGRESOS DEL CAFÉ CONVENCIONAL: UN ANÁLISIS DE PANEL BALANCEADO. PERIODO 1991 - 2010
}

Flor del Carmen Rivera Bocanegra' ${ }^{1}$, Laura Silvia Alvarado Barbaran²

Fecha de recepción: 14-01-13

Fecha de aceptación: 21-08-2013

\section{Resumen}

En el Perú el café convencional representa el cuarto cultivo más importante en el sector agrícola y como todo cultivo es sensible a los cambios del clima afectando o beneficiando su producción. Este estudio tiene como finalidad analizar el impacto del cambio climático sobre la variación de ingresos agrícolas de los productores cafetaleros de las principales zonas de producción en el Perú, lo cual es relevante pues tiene un alcance nacional y no local como en estudios previos. Para ello, se utilizó el enfoque de la función de producción, en base a un modelo econométrico de datos panel, con el objetivo de identificar si la variación de las variables ambientales ante el cambio del clima, podría afectar al cultivo del café convencional de los principales departamentos de la selva alta. Los resultados muestran que existe una relación de convexidad entre la temperatura máxima y la producción de café convencional, es decir, que ante un incremento de la temperatura máxima por encima de su valor opimo dará lugar a un nivel de producción más alto y por ende, los ingresos de los agricultores ca-

1 Economista (UNALM-Perú). Asistente de investigación económica ambiental (UNALM). Dirección postal: MZ N2 LT 3 S.S. JUAN PABLO II (Lima-Perú). Teléfono: (511) 6147134 anexo: 239; e-mail:frivera@lamolijna.edu.pe fetaleros no se verán perjudicados. Estos resultados evidencian que seguir cultivando café convencional a largo plazo será rentable para los agricultores de la zona en estudio, el cual aporta el $1.08 \%$ al valor bruto de la producción del sector agrícola.

Palabras Clave: Impacto del Cambio Climático, café convencional, variación de ingresos y enfoque de la función de producción.

Clasificación JEL: Q54.

\section{Abstract}

In the conventional coffee Peru is the fourth most important crop in the agricultural sector as any crop is sensitive to climate changes affecting or benefiting production. This study aims to analyze the impact of climate change on agricultural income variation coffee growers of the main producing areas in Peru, which is relevant because it has a national scope and local in previous studies. For

2 Máster en Innovación Agraria para el Desarrollo Rural (UNALM-Perú). Profesora Auxiliar a dedicación exclusiva (UNALM). Dirección postal: Jr. Valer 595 C Pueblo Libre (LimaPerú). Teléfono: (511) 6147800 anexo:239; e-mail: lalvarado@ lamolina.edu.pe 
this, we used the approach of the production function, based on the panel data econometric model, in order to identify whether the variation of environmental variables to climate change could affect the cultivation of coffee major conventional high forest departments. The results show that there is a relationship between the maximum temperature convexity and conventional coffee production, that is, to a maximum temperature rise above opimo value will lead to a higher production level and therefore the income of coffee farmers will not be harmed. These results show that conventional coffee continue to cultivate long-term will be profitable for farmers in the study area, which contributes $1.08 \%$ to the gross value of agricultural production.

Keywords: Climate Change Impact, coffee conventional approach of the production function, income variation.

\section{JEL Classification: Q54.}

\section{INTRODUCCIÓN}

En el Perú, el impacto del cambio climático ha tenido gran repercusión, principalmente, en la producción de los cultivos agrícolas, como es el caso del café convencional.

En el año 2013, la producción promedio de café en el Perú representa el 4.2\% del Valor Bruto de la Producción del sector agrícola, ocupando el cuarto entre los principales productos agrícolas ${ }^{3}$ (MINAG, 2009). Dicho culti-

3 Los tres primeros productos agrícolas son: la papa con un $7.6 \%$, alfalfa con un $5.1 \%$ y arroz cascara con $5 \%$ del VBP (MINAG, 2009). vo se produce generalmente en la selva alta, donde se posee las condiciones climáticas más adecuadas para su potencial desarrollo, oscilando la temperatura óptima entre $18^{\circ} \mathrm{C}$ y $22^{\circ} \mathrm{C}$, con niveles mínimo y máximo de 16 ${ }^{\circ} \mathrm{C}$ y $24^{\circ} \mathrm{C}$, respectivamente (MINAG, 20094 y Pro-Amazonia, 2003).

En este contexto, la contribución del presente estudio es evaluar el impacto del cambio climático sobre la producción del café convencional en los principales departamentos de la selva alta del Perú (Amazonas, Cajamarca, Huánuco, Junín, San Martín y Puno), en los cuales se produce el $78.4 \%$ del café convencional del país.

A diferencia de otros estudios que analizaron el impacto del cambio climático en el Perú para un solo departamento, uno de los aportes de este estudio es su alcance nacional. Adicionalmente, constituye una contribución a la literatura en el tema, al aplicar el Enfoque de la Función de Producción utilizando un modelo de datos de panel balanceado, el cual tiene la ventaja de poder utilizar mayor cantidad de información, al interrelacionar datos de corte transversal con series de tiempo. Para tal efecto se usó información de producción, superficie cosechada, precipitación y temperatura. Se consideró como unidad de transversal a cada uno de los departamentos, con veinte datos anuales para el período 1991-2010.

El trabajo se divide en tres partes. En primer lugar, se estimó econométricamente la fun-

\footnotetext{
4 Disponible en http://frenteweb.minag.gob.pe/ sisca/?mod=consulta_cult
} 
ción de producción de café convencional mediante un modelo de panel balanceado. En segundo lugar, se escogió la forma funcional de la función de producción y la relación de las variables climáticas y el nivel de producción del café convencional. Por último, se calculó la variación de ingresos de los agricultores en los principales departamentos de la selva alta para el periodo 2011-2100 bajo un determinado escenario climático.

\section{REVISIÓN DE LITERATURA}

Existen muchos estudios que estimaron los efectos del cambio climático sobre el sector agrícola. Entre los principales tenemos el estudio de Deressa, Hassan y Poonyth (2005) para el caso de la caña de azúcar, en que se obtuvo que cuando la temperatura en verano a menor a $23^{\circ} \mathrm{C}$ disminuyen los ingresos netos por hectárea, sucediendo lo contrario cuando la temperatura supera los $23^{\circ} \mathrm{C}$.

El estudio de Ramírez et al. (2010), el cual forma parte de una serie de documentos elaborados por la Comisión Económica para América Latina y el Caribe (CEPAL), analiza el efecto del cambio climático sobre la agricultura utilizando el Enfoque de la Función de Producción y el Enfoque Ricardiano, para Costa Rica, El Salvador, Guatemala, Honduras, Nicaragua y Panamá; para el caso de Belice sólo utilizaron el primero de los enfoques mencionados.

A pesar de la importancia de evaluar el impacto del cambio climático en el sector agrícola peruano, poco se ha avanzado sobre el tema.
A nivel agregado, Vargas (2009) estimó que al año 2030 el Producto Bruto Interno real sería $6.8 \%$ menor al que se tendría en un escenario sin cambio climático; asimismo encontró que, dada la diversidad de climas que caracteriza al Perú, el impacto del cambio climático sería diferente según el departamento y el sector productivo, siendo uno de los más sensibles el sector agrícola.

A nivel departamental, Torres (2010) realizó un análisis económico del impacto del cambio climático en la producción de mango, limón, café y plátano en la región Piura. Para ello, la autora utilizó una función de producción en donde las variables explicativas fueron temperatura, precipitación y el Fenómeno "El Niño", mediante un análisis de serie de tiempo para el período 1970-2009. Para el caso del café, los resultados indican que existe una relación cóncava entre el rendimiento del producto y los niveles de temperatura; es decir, ante un aumento en la temperatura mínima y máxima, el rendimiento del cultivo aumenta en $41 \%$. En el caso del limón, el aumento es de 51.9\%. Sin embargo, para los casos de mango y plátano, los rendimientos disminuyen en $7 \%$ y $39.86 \%$, respectivamente.

Por su parte, Loyola y Orihuela (2011), evaluaron el costo económico del cambio climático en la agricultura de las regiones Piura y Lambayeque, para el periodo 2010-2100. Para ello, se utilizaron algunos cultivos representativos de ambas regiones, tales como arroz, maíz amarillo, limón, mango, caña de azúcar, plátano y algodón. Utilizando una variante del modelo agronómico, establecieron la re- 
lación de cada uno de estos cultivos con la temperatura y la precipitación y se estimó el cambio del beneficio asociado únicamente a las variaciones en el ingreso de cada cultivo. Los resultados sugieren que el costo del cambio climático es significativo, especialmente a largo plazo. Asimismo, se encontró que el comportamiento entre la temperatura y la producción tienen forma convexa para el caso de limón y plátano en Piura.

De la revisión de literatura se concluye que, salvo para el caso de Piura, no se ha analizado el impacto del cambio climático en el café, lo cual motivó la realización de este estudio, para diferentes zonas productoras de este cultivo, seleccionándose a los departamentos de Amazonas, Cajamarca, Huánuco, Junín, San Martín y Puno, que representan el 78\% de la producción nacional de café, el que es cultivado mayoritariamente por pequeños productores (con menos de 5 has. en promedio), quienes por lo tanto tienen baja capacidad para desarrollar cambios tecnológicos en adaptación al cambio climático.

\section{EL MODELO}

La metodología utilizada en este estudio se sustentó en el modelo agronómico del enfoque de la función de producción, el cual permite estimar los efectos del cambio climático en la agricultura a partir de identificar los valores máximos y mínimos de la temperaturas y los niveles de precipitación, cuyas variaciones respecto a un determinado umbral provocan respuestas beneficiosas o perjudiciales para un cultivo determinado y por ende para los respectivos agricultores. Según, Fleischer y Mendelsohn (2007) la función de producción de un cultivo agrícola se expresa de la siguiente forma:

$$
Q=f(X, Z, M)
$$

Donde $Q$ representa la cantidad producida, $X$ que representa la cantidad de insumos, $Z$ engloba a las variables climáticas (temperaturas máxima y mínima, y precipitación) y M representa la habilidad o capacidad de los agricultores. Mendelsohn, Nordhaus y Shaw (1994), sugieren que las variables climáticas deben ser incluidas en el modelo con una forma funcional cuadrática, para poder determinar la relación existente con el nivel de producción. De esta manera, los coeficientes estimados ayudan a predecir el nivel óptimo de producción ante el impacto del cambio climático. Este análisis fue utilizado en los estudios de Galindo (2009) y Ramirez et al (2010).

Es decir, la ventaja de utilizar una función de producción cuadrática es que permitirá calcular la relación entre el nivel de producción del cultivo (variable dependiente) y los valores óptimos de cada uno de los factores climáticos que determinen su producción (temperatura yo precipitación).

Cabe señalar que al no disponer de series históricas de costos insumos, no hemos utilizado en este estudio el otro enfoque existente, que es el Enfoque Ricardiano, el cual consiste en estimar el valor de la tierra como función del ingreso neto agrícola. 


\section{METODOLOGÍA}

\subsection{Recopilación de información}

Para el desarrollo de la investigación se recopiló información del Ministerio de Agricultura (MINAG) de las variables producción y superficie cosechada de café de los siete principales departamentos cafetaleros del Perú.

Para el caso de las variables climáticas, temperatura y precipitación, la información fue obtenida del Instituto Nacional de Estadística e Informática (INEI). Asimismo, analizar la variación de ingresos, se transformaron los precios al productor de corrientes a reales, utilizándose como deflactor el Índice de Precios al Consumidor del año base 1994, obtenido del INEI.

\subsection{Estimación de la función de producción del café convencional:}

Siguiendo a Mendelsohn, Nordhaus y Shaw (1994), para quienes la relación del nivel de producción y las variables climáticas debería tener una forma de $\mathrm{U}$ invertida, es decir, para determinar si existe una relación de convexidad entre las variables señaladas, se formuló la siguiente ecuación cuadrática5:

$Q_{i, t}=\beta_{0}+\beta_{1} S_{i, t}+\beta_{2} T M_{i, t}+\beta_{3} T M^{2}{ }_{i, t}+\beta_{4} T N_{i, t}$ $+\beta_{5} T^{2}{ }_{i, t}+\beta_{6} P P_{i, t}+\beta_{7} P P_{i, t}^{2}+\mu_{i, t}$

$5 \quad$ No se escogió otras formas funcionales por no satisfacer el objetivo del estudio. Además la función cuadrática se asemeja a la forma funcional que utilizaron estudios anteriores (Ramirez et al, 2010) para determinar el impacto del cambio climático en la agricultura de cultivos específicos y/o otros sectores.
Donde:

$Q_{i, t}=$ Producción (Toneladas) del café convencional en el departamento i para el año t.

$S_{i, t}=$ Superficie cosechada (hectáreas) del café convencional en el departamento i para el año t.

$T M_{i, t}=$ Temperatura máxima $\left({ }^{\circ} \mathrm{C}\right)$ en el departamento i para el año .

$T M^{2}{ }_{i, t}=$ Temperatura máxima $\left({ }^{\circ} \mathrm{C}\right)$ al cuadrado en el departamento i para el año .

$T N_{i, t}=$ Temperatura mínima $\left({ }^{\circ} \mathrm{C}\right)$ en el departamento i para el año .

$T N^{2}{ }_{i, t}=$ Temperatura mínima $\left({ }^{\circ} \mathrm{C}\right)$ al cuadrado en el departamento i para el año .

$P P_{i, t}=$ Precipitación $(\mathrm{mm})$ en el departamento i para el año .

$P P^{2}{ }_{i, t}=$ Precipitación $(\mathrm{mm})$ al cuadrado en el departamento i para el año .

$\mu_{i, t}=$ Error aleatorio.

Se empleó el análisis de la primera derivada para encontrar el valor óptimo de la variable climática y la condición de la segunda derivada, para determinar la relación de concavidad o convexidad entre la producción y las variables climáticas. Utilizando el modelo de datos panel, se esperaba que los signos de los coeficientes de los términos lineales $\beta_{2^{\prime}} \beta_{4^{\prime}} \beta_{6}$ sean positivos. Es decir, que en los primeros años 
el incremento en los niveles de temperatura y precipitación generan un aumento en el nivel de producción del café convencional.

Por su parte, para hallar el nivel óptimo de temperatura, en el cual se maximiza la producción de café convencional, se partió del supuesto que $\beta_{3^{\prime}} \beta_{5}$ y $\beta_{7}$ son negativos. Esto indica que ante cualquier incremento de la temperatura por encima de su valor óptimo dará lugar a un nivel de producción más bajo, por lo tanto la función de producción tomará una forma cóncava. También es posible encontrar que los signos de los coeficientes $\beta_{2}, \beta_{4}, \beta_{6}$ sean negativos y los de $\beta_{3}, \beta_{5}$ y $\beta_{7}$ tengan signos positivos, lo que implicaría una función de producción convexa (Mendelsohn, Nordhaus y Shaw, 1994). Esto significaría que el incremento de la temperatura genera pérdidas en el corto plazo para los agricultores, alcanzando una producción mínima, mientras genera incrementos de la producción a largo plazo.

Siguiendo a Ramírez et al (2010), se formularon las siguientes especificaciones alternativas a ser probadas:

$Q_{i, t}=\beta_{0}+\beta_{1} S_{i, t}+\beta_{2} T M_{i, t}+\beta_{3} T M^{2}{ }_{i, t}+\beta_{4} T N_{i, t}$ $+\beta_{5} T N^{2}{ }_{i, t}+\beta_{6} P P_{i, t}+\beta_{7} P P^{2}{ }_{i, t}+u_{i, t}$

$Q_{i, t}=\beta_{0}+\beta_{1} S_{i, t}+\beta_{2} T M_{i, t}+\beta_{3} T M^{2}{ }_{i, t}+\beta_{4} T N_{i, t}$ $+\beta_{5} T N^{2}{ }_{i, t}+u_{i, t}$

$Q_{i, t}=\beta_{0}+\beta_{1} S_{i, t}+\beta_{4} T N_{i, t}+\beta_{5} T N^{2}{ }_{i, t}+\beta_{6} P P_{i, t}$ $+\beta_{7} P P_{i, t}^{2}+u_{i, t}$

$Q_{i, t}=\beta_{0}+\beta_{1} S_{i, t}+\beta_{4} T N_{i, t}+\beta_{5} T N^{2}{ }_{i, t}+u_{i, t}$
$Q_{i, t}=\beta_{0}+\beta_{1} S_{i, t}+\beta_{2} T M_{i, t}+\beta_{3} T M^{2}{ }_{i, t}+\beta_{6} P P_{i, t}$ $+\beta_{7} P P_{i, t}^{2}+u_{i, t}$

$Q_{i, t}=\beta_{0}+\beta_{1} S_{i, t}+\beta_{2} T M_{i, t}+\beta_{3} T M^{2}{ }_{i, t}+u_{i, t}$

La importancia de definir diferentes alternativas del modelo es poder identificar qué variables son innecesarias o irrelevantes al modelo, lo cual podría ocurrir por la posible correlación entre las variables explicativas, provocando así que los paramentos sean no significativos.

En cuanto al tratamiento de los datos panel, se estimaron seis tipos de ecuaciones para los modelos Pooled y de Efectos Fijos (MEF) y sólo dos ecuaciones para el Modelo de efectos aleatorios (MEA), dado que el número de unidades transversales (departamentos) debe ser mayor al número de variables explicativas ${ }^{6}$. Por lo tanto, para la elección del mejor modelo se tuvo en consideración dos criterios de análisis:

- Se escogió como mejor ecuación aquella que cumplía con los signos adecuados de la teoría económica, es decir que los coeficientes de las variables temperatura y precipitación en términos lineales y cuadráticos tenga signos diferentes, para obtener valores positivos de la temperatura y precipitación. Estos valores además de ser positivos, deben cumplir con los rangos óptimo de temperatura y precipitación sugeridos en el estudio de Pro Amazonia-MINAG (2003).

6 En el Anexo 3, solo se muestran los principales modelos de datos panel las cuales fueron escogidos según los criterios de decisión establecidos en el estudio. 
- Las mejores ecuaciones para cada modelo de estimación, debían tener los valores más bajos de los criterios Akaike y Schwarz. Asimismo, los coeficientes asociados a cada variable regresora debía ser significativos y el valor del $\mathrm{R}^{2}$ ajustado debe ser alto.

Es importante mencionar que en todas las estimaciones, según cada tipo de modelo, se corrigieron problemas de heteroscedasticidad y autocorrelación entre cada unidad transversal utilizando los errores estándar robustos White Cross-Section y Cross-Section Sur, respectivamente.

\subsection{Variación de ingresos en la producción del café convencional}

Por último, para estimar la variación de los ingresos en la producción del café convencional en los principales departamentos de la selva alta, se utilizó las proyecciones de temperatura realizadas por el Instituto Nacional de Investigaciones Espaciales de Brasil (en adelante, INPE, por el original en portugués), para un escenario caracterizado por altos incrementos de dióxido de carbono $\left(\mathrm{CO}_{2}\right)^{7}$, aunque siempre tomando como referencia los estudios del SENAMHI, que establecen la variación anual de temperatura a nivel de las regiones naturales (costa, sierra y selva) subdividas a su vez en norte, centro y sur para los periodos 2010-2030, 2031-2050, y 2051-2100, tal como se muestra en Anexo 2.
Con el fin de obtener mediciones comparativas, se cálculo el Valor Presente Neto de los ingresos anuales con tasas de descuento de $0.5 \%, 2 \%, 4 \%$, sugeridas por Ramírez et al (2010) y Galindo (2009). Adicionalmente, se utilizó la tasa de descuento social de $10 \%$ establecida para el Perú por el Ministerio de Economía y Finanzas (MEF, 2011) ${ }^{8}$. En cualquier caso, se trata de identificar si a largo plazo resulta conveniente o no seguir cultivando café convencional, ante el impacto del cambio climático, hasta el año 2100.

Siendo interés de este trabajo medir el cambio en el ingreso atribuido a la variación climática, se usaron los parámetros estimados del mejor modelo, elegido en base a los criterios de selección ya mencionados anteriormente, y se calculó la producción bajo un contexto de cambio climático $\left(Q_{c c}\right)$ y el nivel de producción sin cambio climático $\left(\mathrm{Q}_{\mathrm{scc}}\right)$ para un determinado periodo.

Asumiendo que el precio del cultivo (en términos reales) permanece constante, expresando IT como el ingreso total y CT como el costo total de producción, para un determinado periodo $t$, la función de beneficio con la que se trabajó fue la siguiente:

$$
B_{i t}=I T\left(Q_{i t}\right)-C T\left(Q_{i t}\right)
$$

Donde las funciones IT y CT dependen del nivel de producción. De esta manera, el cambio en el beneficio es igual a:

$$
\Delta B_{i t}=\Delta I T\left(Q_{i t}\right)-\Delta C T\left(Q_{i t}\right)(4)
$$

$8 \quad$ R.D. No 001-2011-EF/63.01 - Establecido por el Ministerio de Economía y Finanzas (MEF, 2011). 
Luego, asumiendo que los costos totales, en términos reales, permanecen constantes durante el periodo de análisis, entonces el cambio en el beneficio es equivalente únicamente a la variación del ingreso. Por lo tanto la expresión (4) se transforma en:

$$
\Delta B_{i t}=\Delta I T\left(Q_{i t}\right)=P_{i t}^{*} \Delta\left(Q_{i t}\right)
$$

El supuesto que $\Delta C T\left(Q_{i t}\right)=0$, se sustenta en el hecho que en el largo plazo el proceso de aprendizaje les permite a los agricultores obtener no sólo un mayor conocimiento de las técnicas agronómicas, sino también una reducción de procesos y por ende una disminución de costos.

Según la ecuación (5), el cambio en el ingreso total depende de la variación del nivel de producción. En otras palabras, es la diferencia de la producción para un contexto con cambio climático $\left(Q_{C C C}\right)$ para un periodo $(\mathrm{t})$ menos el nivel de producción sin cambio climático $\left(Q_{S C C}\right)$ del año 2010. Asumiendo que el precio del cultivo, en términos reales, permanece constante al año base 2010, entonces el cambio en el ingreso está dado por:

$$
\Delta \mathrm{B}_{\mathrm{it}}=\Delta \mathrm{IT}_{\mathrm{it}}=\overline{\mathrm{P}}_{\mathrm{i} 2010} *\left(\mathrm{Q}_{\mathrm{it}(\mathrm{CCC})}-\overline{\mathrm{Q}}_{\mathrm{i} 2010(\mathrm{SCC})}\right)
$$

En consecuencia, el cambio en el beneficio será equivalente al cambio del ingreso total, el que se calcula multiplicando los precios en chacra reales por el cambio en la producción que se obtendrá si el clima no varía.

La ecuación (6) implica estimar la variación del bienestar ante el efecto de los cambios de tem- peratura y precipitación, mediante el cambio en el ingreso, el cual a su vez, al suponer precios constantes, es igual a la cosecha estimada menos la cosecha proyectada. Esto permite tener aproximaciones de las pérdidas o ganancias a largo plazo para los agricultores de las zonas en estudio, y así determinar si es conveniente o no seguir produciendo café convencional.

Para determinar los ingresos de los agricultores bajo un escenario de cambio climático se empleó las proyecciones de temperatura del INPE (ver Anexo 2). Asimismo, para el cálculo de los ingresos de los agricultores bajo un escenario sin cambio climático se asumió el nivel de producción y el precio real del año 2010 como constantes hasta el año 2100, bajo el supuesto de que no hay ninguna variación (ni incremento ni disminución) de las variables meteorológicas que afectan al café convencional en el largo plazo.

Por otro lado, no se evaluó un segundo escenario bajo el supuesto de precios en chacra reales proyectados, debido que ello requeriría asumir grandes supuestos sobre cómo cambiaría el mercado mundial frente al cambio climático, lo cual aumentaría el nivel de incertidumbre de la estimación. Por ello, resultó más prudente asumir que el comportamiento de estos precios permanezcan constantes, tal como lo sugieren Sanghi y Mendelsohn (2008).

Asimismo, este escenario alternativo sobreestimaría los ingresos de los agricultores, dado que el alto nivel de incertidumbre sobre el comportamiento de precios, en este tipo de estudios, conduce a obtener resultados muy positivos (grandes beneficios) o muy negati- 
vos (grandes pérdidas). Por tal motivo, al asumir precios reales constantes se obtiene una evolución más atenuada del nivel de precios, acorde con las proyecciones del nivel de temperatura (INPE, 2011). No obstante, es necesario reconocer que al asumir precios reales constantes cabría la posibilidad de sobrestimación o subestimación de ingresos, si bien el sesgo sería más moderado.

\section{RESULTADOS}

Los resultados muestran que los modelo POOLED y $\mathrm{MEA}^{9}$, son los que cumplen con los criterios de decisión y se encuentran dentro del rango del nivel de temperatura óptima señalado por Pro Amazonia (2003) de 18 a $22^{\circ} \mathrm{C}$ con valores mínimo y máximo de 16 y $24^{\circ} \mathrm{C}$, respectivamente, que es considerado adecuado para un buen crecimiento y desarrollo del cultivo en la selva alta.

Luego, se procedió a realizar el test de Breusch-Pagan para escoger el mejor modelo entre el POOLED y el MEA, lo cual se muestra en el Anexo 5. Los resultados indican que no se rechaza la hipótesis nula de Breusch y Pagan; es decir, no existe diferencias entre cada unidad trasversal del modelo POOLED y del MEA, a través del tiempo, por lo que se concluye que el MEA no es apropiado y, en consecuencia, se escoge como mejor al modelo Pooled, el cual representará la función de producción del café convencional.

La interpretación del mejor modelo es que no existe diferencias entre cada uno de los

9 Ver Anexos 3 y 4. principales departamentos cafetaleros de la selva alta en el periodo 1991-2010, lo cual es consistente con la cercanía geográfica de los productores.

La resultante función de producción del café convencional tiene la siguiente ecuación:

$Q_{i, t}=17274.41+0.76 S_{i, t}-1742.32 T M_{i, t}$ $+39.25 \mathrm{TM}_{i, t}^{2}+\mu_{i, t}$

La expresión (7) evidencia que la temperatura máxima es la variable climática que determina el nivel de producción del café convencional en los principales departamentos de la selva alta. Asimismo, el resultado permite comprobar que existe una relación directa entre la producción de café convencional y la superficie cosechada. Es decir, que un aumento de la superficie cosechada ocasionará un incremento en el nivel de producción del café.

Por otro lado, el signo negativo de la temperatura máxima en términos lineales, significa que en los primeros años los incrementos en los niveles de temperatura provocan una disminución en el nivel de producción del café. Por el contrario, para la temperatura máxima en términos cuadráticos se observó un signo positivo, lo que indica que a largo plazo, al sobrepasar un umbral (valor óptimo), la temperatura máxima ocasionará incrementos en los niveles de producción.

Debe señalarse que no fue considerada la ecuación que incluye como variables explicativas a la temperatura mínima y a la precipitación, porque el nivel de la temperatura no 
estaba dentro del rango establecido por Pro Amazonia (2003), además que los valores de los coeficientes no son estadísticamente significativos. Algo similar ocurrió con la variable precipitación. Estos resultados son consistentes con el estudio de Kabubo y Karanja (2007), en el que se reconoce que los cambios en la temperatura son, en general, más importantes que los cambios en la precipitación, así como con los estudios de Ramírez et al (2010) y Torres (2010). Otra forma de justificar que la temperatura máxima se encuentre en la función de producción, es que el cultivo del café se adapta mejor a las altas temperaturas que caracteriza a las zonas tropicales. Asimismo, el nivel de temperatura óptima se encuentra dentro del rango establecido por Pro Amazonia y cumple con los criterios del estudio.

Cabe indicar que existe la posibilidad de incorporar la variable climática precipitación en este tipo de estudios, utilizando data trimestral o mensual. Sin embargo, no pudimos incorporar dicha variable en dicho estudio, por no contar con la información disponible para los periodos de análisis. Por ello, el presente estudio es una mejor aproximación para analizar y explicar el impacto del cambio climático en el café convencional ante un incremento de la variable temperatura máxima.

\subsection{Optimización de la} función de producción del
café convencional y valor
óptimo de la variable
climática

En primer lugar, se analizó la ecuación (7), me- diante la condición de la primera derivada, encontrándose que el valor óptimo de la temperatura máxima es de $22.20^{\circ} \mathrm{C}$, valor que es el mismo para todos los principales departamentos cafetaleros, debido a que se escogió el mejor Modelo Pooled, el cual omite las diferencias que puedan existir entre cada unidad transversal (principales departamentos cafetaleros de la selva alta) a través del tiempo.

En segundo lugar, se calculó la segunda derivada para encontrar la relación de concavidad o convexidad entre la producción y la variable climática (temperatura máxima), tal y como se muestra en el Anexo 6. Los resultados arrojaron un valor positivo de 78.49358 , lo que quiere decir que existe una relación de convexidad entre la producción del café convencional y la temperatura máxima, es decir, un incremento de la temperatura máxima, luego de obtenido su valor optimo, dará lugar a un nivel de producción más alto, por lo que se concluye que la función de producción tiene forma convexa, tal como se muestra en la figura 1.

La convexidad reflejada en la figura 1, también permite apreciar que mientras a corto plazo se obtiene una producción mínima de 21.80 miles de TM, cuando la temperatura llega a su nivel óptimo, a largo plazo será beneficioso obtener un mayor nivel de producción de café convencional.

La forma convexa de la función de producción del café convencional concuerda con la teoría y con estudios anteriores, como el de Mendelsohn, Nordhaus y Shaw (1994), quienes afirman que es posible encontrar que los signos 
Figura 1. Producción de café convencional vs. Temperatura máxima

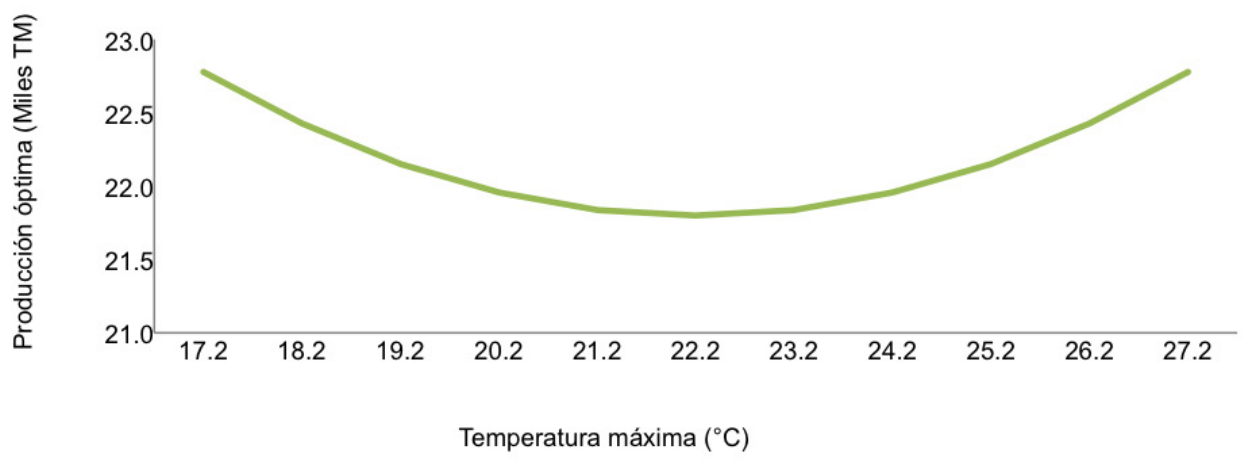

Fuente: Elaboración Propia.

de los coeficientes en términos lineales de la variable climática (temperatura máxima) sea negativo y los coeficientes en términos cuadráticos de la misma variable arrojen signo positivo, en este caso la función de producción será de forma convexa. Es decir, que el incremento de la temperatura genera pérdidas en el corto plazo para los agricultores llegando a una producción mínima y en el largo plazo incrementos en la temperatura conllevará a incrementos en la producción. Estos resultados también son congruentes con el estudio de Deressa, Hassan y Poonyth (2005), así como en Loyola y Orihuela (2011).

Finalmente, la relación convexa entre la producción de un cultivo agrícola y la temperatura, implica que el impacto del cambio climático no necesariamente tiene efectos adversos para todo tipo de cultivo, ya que ello depende tanto del tipo de región como del tipo de cultivo (Gerald, 2009). Para el caso del café convencional le favorece principalmente temperaturas altas, por ser un producto de zona tropical, donde se obtiene una mejor calidad de café en el país.

\subsection{Variación de ingresos del café convencional en los principales departamentos de la selva alta}

Se tomó como referencia las estimaciones realizadas por el INPE para los periodos 20102030, 2031-2050 y 2051-2100, de la variación de temperatura para uno de los escenarios más extremos, denominado $A 2^{10}$, según ubicación geográfica de los principales departamentos que cultivan el café convencional: Selva alta Norte (Amazonas, Cajamarca y San Martin); Selva alta Central (Huánuco y Junín) y Selva alta Sur (Puno).

Asimismo, para todos los departamentos en estudio se utilizaron las tasas de descuento sugeridas por Ramírez et al (2010) y Galindo (2009): 0.5\%, 2\%, y 4\%. Adicionalmente, los

10 Los escenarios de emisiones globales del Panel Intergubernamental de Cambio Climático (IPCC, 2007) son: A1, $A 2, B 1 y B 2$. El escenario $A 2$ describe un mundo heterogéneo con una población mundial en continuo crecimiento económico por habitante y un proceso de cambio tecnológico de manera fragmentada. Asimismo, se considera a este escenario como el más extremo por presentar mayor nivel de contaminación de $\mathrm{CO}_{2}$. 
Figura 2. Producción de café convencional estimado para el año 2100

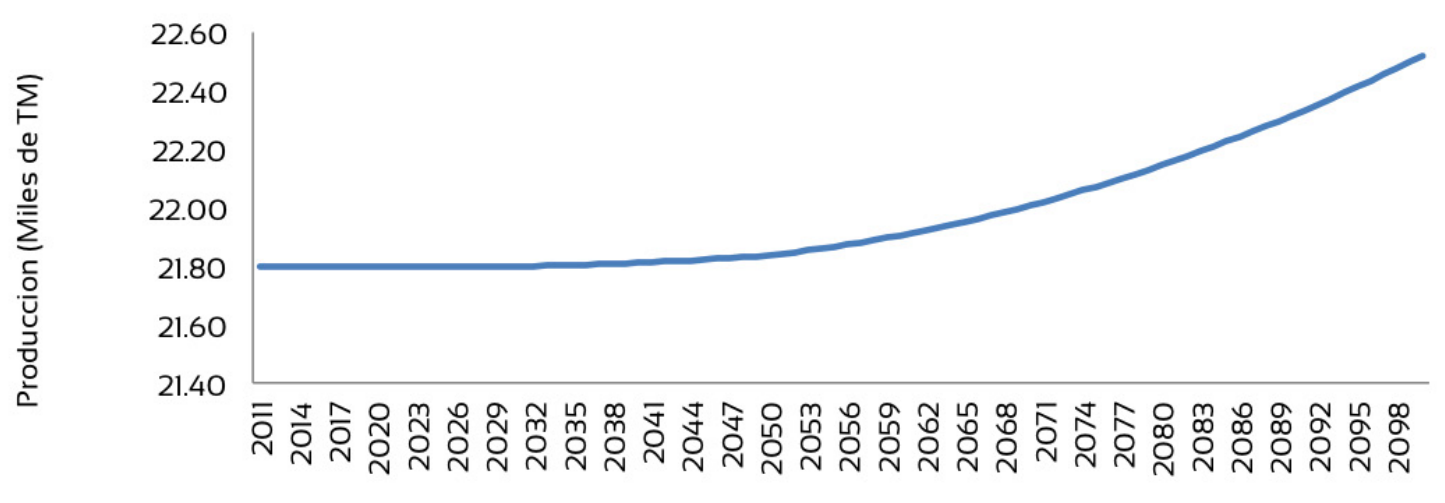

Años

Fuente: Elaboración Propia.

resultados se compararon con la tasa de descuento social de $10 \%$, utilizada en el Perú.

En la figura 2, se observa que a partir del año 2011 se alcanzó un nivel mínimo de producción de 21.80 Miles de TM y a partir del año 2031 se dará un incremento más fuerte o más notorio de la producción hasta el año 2100 con una producción promedio de 22.52 Miles de TM.

\subsection{Proyecciones de la variación de ingresos causado por el aumento de la temperatura máxima}

En la tabla 1, se muestra los resultados del cambio en los ingresos de los agricultores, como consecuencia de la variación del nivel de temperatura máxima en la producción del café convencional.

Se observa que en todos los departamentos la variación de los ingresos sigue la forma del modelo de regresión escogido (POOLED) para el cultivo del café convencional. Asimismo, al analizar cada departamento en un mismo intervalo de tiempo, sus ingresos disminuyen cuando el valor de la tasa de descuento aumenta, es decir que los agricultores de los departamentos seguirán produciendo café convencional, ya que para éstas tasas de descuento, a pesar que se genera menos ingresos, éstos siguen siendo positivos.

Por ello, a los agricultores les favorece que el comportamiento de la tasa de descuento sea constante en el tiempo ya que obtendrán mayores ingresos, de lo contrario deberán estar a la expectativa de que las tasas de descuento no varíen drásticamente porque sus ingresos futuros se verán afectados reduciéndose lentamente. Es decir, que ante un incremento de la temperatura, los ingresos de los agricultores aumentarán para unas mismas tasas de descuento cumpliendo también con la relación y criterio de convexidad que se obtuvo al analizar la variable temperatura máxima 


\begin{tabular}{|c|c|c|c|c|c|c|}
\hline \multirow[t]{2}{*}{ Región } & \multirow{2}{*}{$\begin{array}{l}\text { Departa- } \\
\text { mentos }\end{array}$} & \multirow[t]{2}{*}{ Años } & \multicolumn{4}{|c|}{$\begin{array}{c}\text { Variación de ingresos para cada tasa de des- } \\
\text { cuento (S/.) }\end{array}$} \\
\hline & & & $0.50 \%$ & $2 \%$ & $4 \%$ & $10 \%$ \\
\hline \multirow{9}{*}{ Selva alta Norte } & \multirow{3}{*}{ Amazonas } & $2011-2030$ & 696 & 556 & 416 & 187 \\
\hline & & $2031-2050$ & 19,323 & 11,819 & 6,288 & 1,133 \\
\hline & & $2051-2100$ & 713,843 & 248,441 & 67,304 & 2,915 \\
\hline & \multirow{3}{*}{ Cajamarca } & $2011-2030$ & 715 & 571 & 427 & 192 \\
\hline & & $2031-2050$ & 20,708 & 12,654 & 6,723 & 1,204 \\
\hline & & $2051-2100$ & 779,145 & 270,947 & 73,284 & 3,143 \\
\hline & \multirow{3}{*}{ San Martín } & $2011-2030$ & 513 & 410 & 307 & 138 \\
\hline & & $2031-2050$ & 14,244 & 8,712 & 4,635 & 835 \\
\hline & & $2051-2100$ & 526,223 & 183,143 & 49,615 & 2,148 \\
\hline \multirow{6}{*}{ Selva alta Central } & \multirow{3}{*}{ Huánuco } & $2011-2030$ & 651 & 520 & 389 & 175 \\
\hline & & $2031-2050$ & 19,503 & 11,910 & 6,320 & 1,126 \\
\hline & & $2051-2100$ & 664,534 & 232,424 & 63,487 & 2,826 \\
\hline & \multirow{3}{*}{ Junín } & $2011-2030$ & 570 & 460 & 349 & 163 \\
\hline & & $2031-2050$ & 20,080 & 12,370 & 6,632 & 1,205 \\
\hline & & $2051-2100$ & 713,976 & 250,975 & 69,111 & 3,146 \\
\hline \multirow{3}{*}{ Selva alta Sur } & \multirow{3}{*}{ Puno } & $2011-2030$ & 1,080 & 862 & 645 & 290 \\
\hline & & $2031-2050$ & 20,779 & 12,828 & 6,924 & 1,324 \\
\hline & & $2051-2100$ & 665,678 & 233,141 & 63,976 & 3,014 \\
\hline
\end{tabular}

Fuente: elaboración propia.

versus el nivel de producción en base a la función de producción del café convencional.

\section{CONCLUSIONES Y RECOMENDACIONES}

\subsection{Conclusiones}

El impacto del cambio climático sobre los ingresos del café convencional en los principales departamentos de la selva alta, no necesariamente provoca una disminución de los niveles de ingreso. Sin embargo, esto depende principalmente del comportamiento de los precios. Los resultados muestran que es rentable seguir cultivando café convencional para los agricultores de la zona en estudio, puesto que obtendrán ganancias a largo plazo. Asimismo, dicho incremento en el nivel ingresos aporta el $1.08 \%$ al Valor Bruto de la Producción del Sub Sector agrícola.

Se puede afirmar entonces que el impacto del cambio climático es favorable para el cultivo del café convencional en los principales departamentos de la selva alta peruana, lo cual coincide con los resultados de otros estudios previos realizados en la región Piura. De esta manera, se sustenta que el impacto del cambio climático no necesariamente tendrá efectos adversos para todos los cultivos 
del Perú, ya que ello depende de la región y tipo de cultivo. (Gerald et al, 2009). Particularmente, para el caso del café convencional, por ser un producto de zonas tropicales, es favorecido por altas temperaturas, lo que permite obtener un producto de buena calidad.

De esta manera, se concluye que resulta beneficioso para los agricultores de los principales departamentos de la selva alta seguir cultivando café convencional, con su correspondiente contribución de $1.08 \%$ al incremento en el Valor Bruto de la Producción agrícola del país.

Debe también señalarse que estudios sobre el impacto del cambio climático están sujetos a ciertas limitaciones, ya que los resultados podrían relativizarse por la extensión del período analizado, por la validez de los datos utilizados, así como por el carácter incierto de ciertas variables, como los precios al productor. Por este motivo, en este estudio utilizamos el supuesto de precios reales al productor, constantes en el tiempo, evitando así el tener que proyectar para un período tan extenso. Otro tipo de dificultades afrontadas se refiere a la obtención de información precisa del clima de una determinada zona. En este aspecto, se ha supuesto, por simplificación, que los datos de determinadas estaciones meteorológicas corresponden a las localizaciones geográficas que se estudian, lo cual evidentemente implica ciertos riesgos de inexactitud. Adicionalmente, se ha empleado información sólo de un escenario climatológico, lo cual limita aun más los resultados obtenidos.
Por ello es importante mencionar que los resultados encontrados en el estudio deben ser tomados con mucha cautela ya que son parte de un proceso reflexivo y metodológico, dentro de una perspectiva integral del análisis del impacto del cambio climático, enfocado en este caso particular a los ingresos de los productores de café convencional en la selva alta del Perú. Adicionalmente, tomando como punto de partida estudios como el nuestro, se deben realizar mayores acciones preventivas que permitan la adaptación al cambio climático de cultivos de importancia nacional y que involucra a los agricultores de las zonas en estudio.

\subsection{Recomendaciones}

El problema principal afrontado en ésta investigación, fue no contar con información del costo de producción del cultivo del café convencional. Debido a ésta limitación, al analizar los beneficios de los agricultores, se consideró que los costos son constantes, lo que implicó asumir que a largo plazo el proceso de aprendizaje del agricultor le permite un mayor conocimiento sobre las alternativas, técnicas y distribución agronómicas, con el fin de enfrentar las variaciones del clima y reducir los costos a incurrir.

La limitación anteriormente expuesta, también impide aplicar en este tipo de estudios, el Enfoque Ricardiano. En general, por todo lo mencionado, recomendamos la aplicación de encuestas para determinar los costos de producción de productos como el analizado. Complementariamente, creemos que las instituciones encargadas (Ministerio de Agri- 
cultura, Instituto Nacional de Estadísticas, etc.), deberían realizar censos agropecuarios con mayor frecuencia, para así estimar estos costos y favorecer la realización de investigaciones similares.. Asimismo, se recomienda hacer un análisis por empresas productoras de café, para tener un contraste de cómo afecta el cambio climático en la producción de café para estas empresas y establecer medidas de adaptación y mitigación mediante la información obtenida utilizando un Enfoque Ricardiano.

\section{AGRADECIMIENTOS:}

Los autores desean agradecer a los dos dictaminadores anónimos por sus observaciones que ayudaron a enriquecer este manuscrito así como a los economistas José Luis Nolazco Cama y Miguel Ángel Alcántara por sus valiosos comentarios. 


\section{REFERENCIAS BIBLIOGRÁFICAS}

Baltagi, B.H. (2008). Econometric Analysis of Panel Data (4a Ed.). John Willey and Sons Ltd. Inglaterra.

Barco, D. y Vargas, P. (2010). “El Cambio Climático y Sus Efectos en el Perú", en Moneda. No. 143, pp. 25-29. Banco Central de Reserva del Perú.

Beltrán, A. y Castro, J.F. (2010). Modelos de datos de panel y variables dependientes limitadas: teoría y práctica. Universidad del Pacífico. Lima.

Cámara Peruana del Café y Cacao -CPCC (2007). El café en el Perú. Lima.

Cline, W.R. (2007). Global warming and agricultural: impact estimates by country. Center for Global Development and Peterson Institute for International Economics. Washington, D.C.

Dell, M., Jones, B.F. y Olken, B.A. (2008). Climate Change and Economic Growth: Evidence from the last half century. Working Paper 14132. National Bureau of Economic Research. Junio.

Deressa, T., Hassan, R.M. y Poonyth, D. (2005). "Measuring the Impact of Climate Change on South African Agriculture: the case of sugarcane Growing regions". Agrekon. Vol 44(4), pp. 524-542.

Fleischer, A., Lichtman, I. y Mendelsohn, R.
(2007). Climate Change, Irrigation, and Israeli Agriculture. World Bank, Policy Research Working Paper, No. 4135.

Galindo, L.M. (2009). Economía del Cambio Climático en México.UNAM, México.

Green, W. (2002) Econometric Analysis. Prentice Hall. (5a Ed.). New York University.

Instituto Nacional de Estadística e Informática del Perú - INEI. http://www1.inei.gob.pe/ web/aplicaciones/siemweb/.

Instituto Nacional de Pesquisas Espaciais de Brasil - INPE. ftp://ftp.cptec.inpe.br/modelos/ clima/RECCS/Brasil

IPCC. (2007) Climate Change: Fourth Assessment Report of the Intergovernmental Panel on Climate Change. Cambridge University Press. Cambridge.

Junta Nacional de Cafetaleros del Perú - JNC. http://www.juntadelcafe.org.pe/

Kabubo, J. y Karanja, F (2007) The Economic Impact of Climate Change on Kenyan Crop Agriculture: Ricardian Approach. Policy Research Working, Paper 4334, The World Bank.

Loyola R. (2009). Los Costos del Cambio Climático en el Perú. Guión propuesto para los Estudios Nacionales de la Economía Del Cambio Climático en Sudamérica (ERECC-SA). Ministerio del Ambiente.

Loyola R. y Orihuela C. (2011). El costo econó- 
mico del cambio climático en la agricultura peruana: El caso de la región Piura y Lambayeque. PMP13-2009. Consorcio de Investigación Económica y Social-CIES. Disponible en: www.cies.org.pe

Mendelsohn, R., W. Nordhaus y D. Shaw. (1994). "The Impact of Global Warming on Agriculture: A Ricardian Analysis". American Economic Review (84), pp.753-771.

Ministerio de Agricultura - MINAG. (2010). Dinámica agropecuaria. 1997 -2009. Oficina de Estudios Económicos y Estadísticos.

Ministerio de Agricultura del Perú - MINAG. www.minag.gob.pe

Ministerio del Ambiente del Perú y Servicio Nacional de Meteorología e Hidrología del Perú - MINAM- SENAMHI (2008). Escenarios Climáticos en el Perú para el Año 2030.

Nelson, G.C., et al (2009). El cambio climático. El impacto en la agricultura y los costos de adaptación. Instituto Internacional de Investigación sobre Políticas Alimentarias IFPRI Washington, D.C.

Pro-Amazonia - MINAG. (2003). Programa para el Desarrollo de la Amazonía: Caracterización del Café. Lima.

Ramirez, D. et al (2010) Istmo Centroamericano: Efectos del Cambio Climático Sobre la Agricultura. CEPAL.

Sanghi A. y Mendelsohn R. (2008). "The im- pacts of global warming on famers in Brazil and India". Global Environmental Change , $\mathrm{N}^{\circ} 18$, pp 655-665.

Seo, S.N. y Mendelsohn, R. (2008). "An Analysis of Crop Choice: Adapting to climate Change in Latin American Farms". Ecological Economics, 67, pp. 109-116.

Torres Ruiz, L. (2010). Análisis Económico del Cambio Climático en la Agricultura de la Región Piura-Perú, Caso: Principales Productos Agroexportables. CIES. Universidad Nacional de Piura.

Vargas, P. (2009). El Cambio Climático y sus Efectos en el Perú. Banco Central de Reserva del Perú - Documento de Trabajo №2009-14. 


\section{ANEXOS}

\section{Anexo 1. Descripción de las variables}

\begin{tabular}{|c|c|c|c|c|}
\hline Variable & Símbolo & Definición & $\begin{array}{l}\text { Unidad } \\
\text { de } \\
\text { medida }\end{array}$ & $\begin{array}{c}\text { Tipo de } \\
\text { variable }\end{array}$ \\
\hline Unidades temporales & $\mathrm{t}$ & Intervalo de tiempo de 1991 hasta 2010 & Año & Discreta \\
\hline Unidades transversales & $\mathrm{i}$ & $\begin{array}{l}\text { Principales departamentos cafetaleros de la selva } \\
\text { alta: Amazonas, Cajamarca, Huánuco, Junín, San } \\
\text { Martín y Puno. }\end{array}$ & & $\begin{array}{c}\text { Cualitativa } \\
\text { nominal }\end{array}$ \\
\hline Dependiente & Q & Producción de café convencional & Ton. & $\begin{array}{c}\text { Cuantitativa } \\
\text { continua }\end{array}$ \\
\hline Independiente & $\begin{array}{l}S \\
P \\
T M \\
\text { TN } \\
\text { PP }\end{array}$ & $\begin{array}{l}\text { Superficie Cosechada del café convencional } \\
\text { Precio chacra real café convencional (base=1994) } \\
\text { Temperatura máxima } \\
\text { Temperatura mínima } \\
\text { Precipitación }\end{array}$ & $\begin{array}{l}\text { Ha. } \\
\text { S/./Ton. } \\
{ }^{\circ} \mathrm{C} . \\
{ }^{\circ} \mathrm{C} . \\
\mathrm{mm} .\end{array}$ & $\begin{array}{c}\text { Cuantitativa } \\
\text { continua }\end{array}$ \\
\hline
\end{tabular}

Fuente: Instituto Nacional de Estadística e Informática (INEI), MINAG, SENAMHI.

Elaboración Propia.

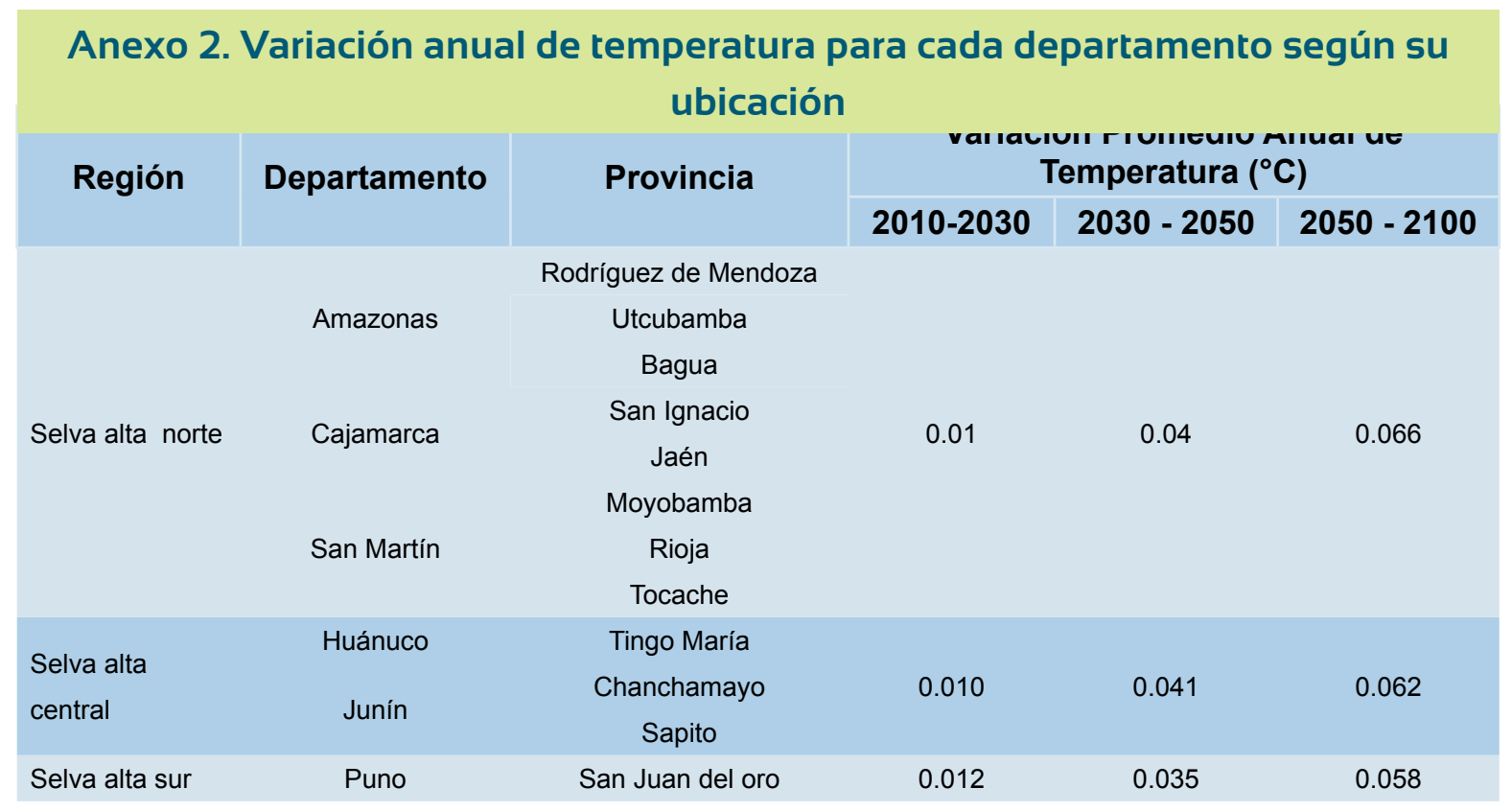

Fuentes: SENAMHI, INPE y Pro Amazonia.

Elaboración Propia. 


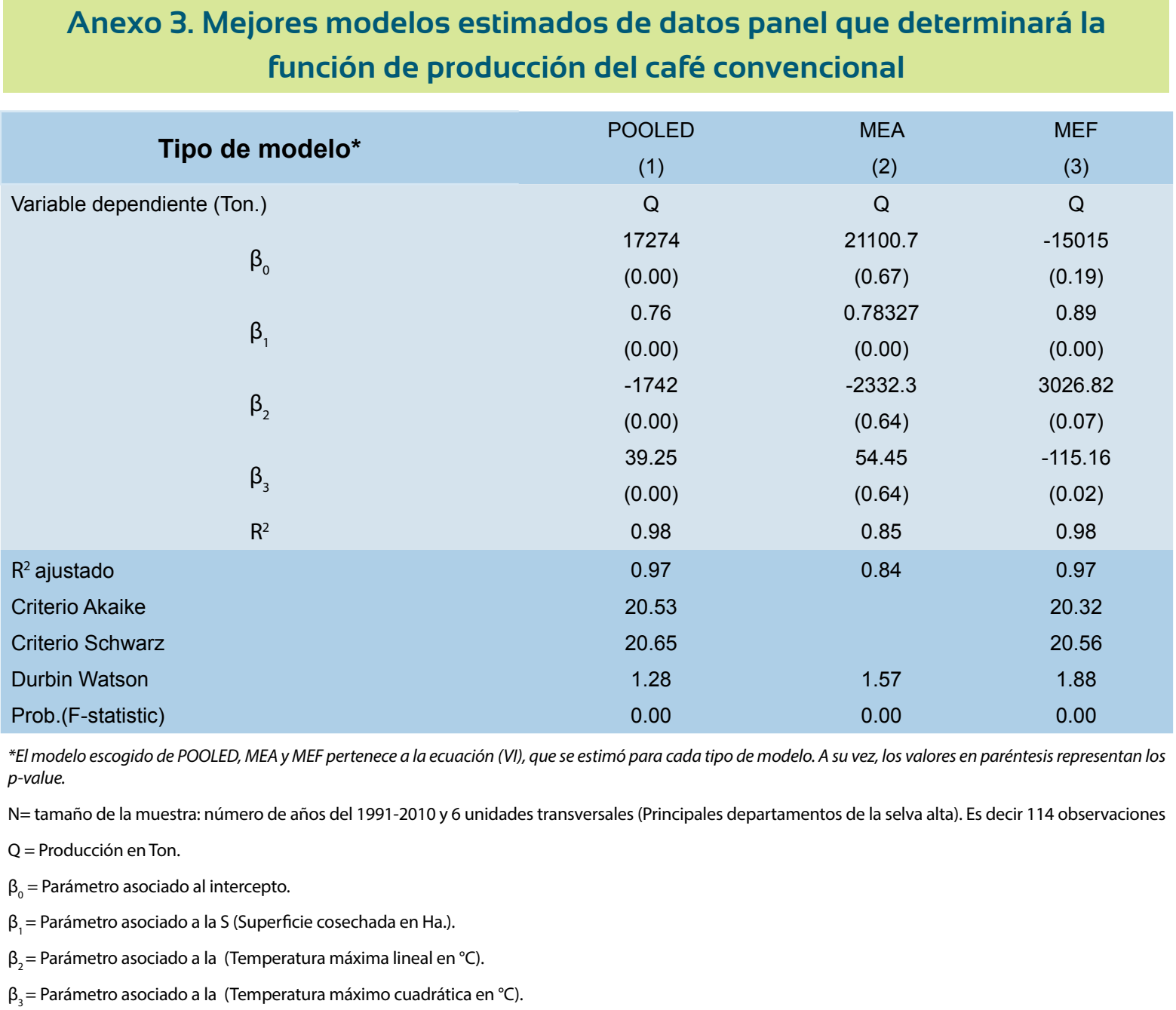

Fuente: Elaboración Propia.

\section{Anexo 4. Nivel de Temperatura óptima para cada tipo de modelo escogido}

\begin{tabular}{|c|c|c|c|}
\hline \multicolumn{1}{|c|}{ Tipo de modelo } & POOLED (1) & MEA (2) & MEF (3) \\
\hline${ }^{*}$ Temperatura Máxima optima $\left({ }^{\circ} \mathrm{C}\right)$ & 22.19 & 21.42 & 13.14 \\
\hline
\end{tabular}

*Variable climática independiente.

Fuente: Elaboración Propia. 


\section{Anexo 5. Test de Breusch-Pagan}

\begin{tabular}{c|c} 
Valor Estadístico & Probabilidad \\
\hline 1.061853 & 0.302793
\end{tabular}

Fuente: Elaboración Propia.

\section{Anexo 6. Relación de la producción del café convencional entre las variables climáticas, según el mejor modelo}

\begin{tabular}{|l|c|c|c|}
\hline Variable climática & Valor óptimo' & $\begin{array}{c}\text { Relación de la } \\
\text { Segunda } \\
\text { derivada }\end{array}$ & $\begin{array}{c}\text { Reducción }\left(\mathbf{Q}_{\mathrm{t}}\right) \mathbf{y} \\
\text { produch } \\
\text { temperatura máxima } \\
\left(\mathbf{T M}_{\mathrm{t}}\right)\end{array}$ \\
\hline Temperatura Máxima $\left(\mathrm{TM}_{\mathrm{t}}\right)$ & $\mathrm{TM}_{\mathrm{t}}{ }^{*}=\frac{-(-1742.32)}{2(39.25)}=22.20$ & $2(39.25)>0$ & Convexa \\
\hline
\end{tabular}

'Se encontró el valor óptimo al igualar a cero la primera derivada.

Fuente elaboración Propia 